\title{
High slope efficiency and low threshold in a diode-pumped epitaxially grown $\mathrm{Yb}$ : YAG waveguide laser
}

\author{
D. Pelenc ${ }^{\mathrm{a}, 1}$, B. Chambaz ${ }^{\text {a }}$, I. Chartier ${ }^{\text {a }}$, B. Ferrand ${ }^{\mathrm{a}}$, C. Wyon ${ }^{\mathrm{a}}$, D.P. Shepherd ${ }^{\mathrm{b}}$, \\ D.C. Hanna ${ }^{b}$, A.C. Large ${ }^{b}$, A.C. Tropper ${ }^{b}$ \\ ${ }^{a}$ LETI (CEA, Technologies Avancées), Grenoble, France \\ ' Optoelectronics Research Centre, University of Southampton, Highfield, Southampton. UK
}

Received 15 August 1994

\begin{abstract}
We report the $1.03 \mu \mathrm{m}$ and $1.05 \mu \mathrm{m}$ laser operation of a diode-pumped Yb-doped YAG planar waveguide. The fabrication and characterisation of such multilayer planar waveguides by liquid phase epitaxy are also described. Diode-pumped laser results ( $\leq 43 \mathrm{~mW}$ threshold, $\geq 77 \%$ slope efficiency) obtained with $2.1 \%$ output coupling compare very favourably with results reported elsewhere for bulk lasers.
\end{abstract}

\section{Introduction}

The development of miniaturised diode-pumped solid state laser systems continues to be an area of intense activity. Low threshold and high slope efficiency are important features of such devices and slope efficiencies of $69 \%$ and thresholds of $5 \mathrm{~mW}$ have already been reported in neodymium-doped systems [1]. In this context ytterbium doped materials offer several advantages as pointed out by Lacovara et al. [2]. The existence of only one manifold of excited $\mathrm{Yb}$ states precludes excited-state absorption processes and also suppresses concentration quenching. Furthermore, with a reduced energy difference between the pump and emitted photon compared to diode-pumped $\mathrm{Nd}$ lasers there is a significantly decreased thermal load. Also, in the case of $Y b: Y \Lambda G$, the large absorption linewidth (the broadest peak situated at $941 \mathrm{~nm}$ and

\footnotetext{
'Present address: Crismatec. Gières, France.
}

another at $968 \mathrm{~nm}$ ) makes it well-suited to diodepumping.

The main drawback of the energy level structure of $\mathrm{Yb}$ is its quasi-three-level nature, a significant part $(5 \%)$ of the total ground state population being in the lower laser level [2], giving rise to a rather high threshold. One way of decreasing the threshold is the use of a guided structure in which high pump power densities are achievable for modest pump power.

Liquid phase epitaxy (LPE) is a promising technique for producing low-loss planar waveguide lasers. This method has already achieved good results in a multimode Nd:YAG waveguide where losses as low as $0.05 \mathrm{~dB} / \mathrm{cm}$ were obtained and a submilliwatt threshold measured [3]. Moreover, this technique allows the realisation of a wide variety of waveguides by controlling the refractive indices and thicknesses of the epitaxial layers. Refractive index differences up to $4.8 \times 10^{-2}$ and thicknesses between 3 and $120 \mu \mathrm{m}$ have been produced. Results on the $946 \mathrm{~nm}$ transition of $\mathrm{Nd}$ : YAG have confirmed the potential of LPE wave- 
Table 1

Composition and properties of several $\mathrm{Yb}$ doped Y AG layers

\begin{tabular}{|c|c|c|c|c|c|c|c|c|c|}
\hline \multicolumn{4}{|c|}{ Growth conditions } & \multicolumn{3}{|c|}{ Layer composition } & \multicolumn{3}{|c|}{ Layer properties } \\
\hline Melt & $\begin{array}{l}T_{\mathrm{s}} \\
\left({ }^{\circ} \mathrm{C}\right)\end{array}$ & $\begin{array}{l}T_{(;} \\
\left({ }^{\circ} \mathrm{C}\right)\end{array}$ & $\begin{array}{l}V_{\mathrm{G}} \\
(\mu \mathrm{m} / \mathrm{mn})\end{array}$ & $\begin{array}{l}X_{Y h} \\
\text { (at.\%) }\end{array}$ & $\begin{array}{l}X_{\mathrm{I} . \mathrm{u}} \\
\text { (at.\%) }\end{array}$ & $\begin{array}{l}X_{\mathrm{Ga}} \\
\text { (at.\%) }\end{array}$ & $\begin{array}{l}\Delta a \\
\left(10^{-3} \AA\right)\end{array}$ & $\begin{array}{l}\Delta a_{\mathrm{c}} \\
\left(10^{-3} \AA\right)\end{array}$ & $\begin{array}{l}\Delta n \\
\left(10^{-3}\right)\end{array}$ \\
\hline A & 1000 & 970 & 1.15 & $3.5 \pm 0.2$ & 0 & 0 & $+4 \pm 0.2$ & $+4.5 \pm 0.2$ & \\
\hline B & 1000 & $\begin{array}{l}970 \\
990\end{array}$ & $\begin{array}{l}1.34 \\
0.4\end{array}$ & $8 \pm 0.2$ & $\begin{array}{l}0 \\
0\end{array}$ & 0 & $+6.6 \pm 0.2$ & $+7.6 \pm 0.2$ & $<1$ \\
\hline $\mathrm{C}$ & 1020 & 1000 & $\begin{array}{l}0.4 \\
1.25\end{array}$ & $\begin{array}{l}7 \pm 0.2 \\
7 \pm 0.2\end{array}$ & $22.5 \pm 0.5$ & $10 \pm 0.5$ & $-0.5 \pm 0.2$ & $\begin{array}{l}+1.9 \pm 0.2 \\
-0.8 \pm 0.2\end{array}$ & $10 \pm 0.5$ \\
\hline $\mathrm{D}$ & 1020 & 1000 & 1.18 & $7 \pm 0.2$ & $23 \pm 0.5$ & $12 \pm 0.5$ & $-5.5 \pm 0.2$ & $-6 \pm 0.2$ & $12 \pm 0.5$ \\
\hline $\mathrm{E}$ & 1030 & 1020 & 0.2 & $7 \pm 0.2$ & $28 \pm 0.5$ & $12 \pm 0.5$ & $-0.5 \pm 0.2$ & $-1.5 \pm 0.2$ & $12 \pm 0.5$ \\
\hline
\end{tabular}

guides for quasi-three-level lasers [4]. Yb:YAG planar waveguide lasers have previously been made by ion-implantation [5] but the propagation losses were quite high at $\sim 2 \mathrm{~dB} / \mathrm{cm}$.

We report here the LPE growth and laser operation of an Yb-doped guide. This planar waveguide was composed of an active layer, with a controlled refractive index, epitaxially grown on a pure YAG substrate. Then, a cladding layer of pure YAG was grown on top in order to have a symmetric index profile and to provide a buried (and hence protected) guiding interface.

\section{Liquid phase epitaxial growth}

The advantages offered by the compositional variations and range of refractive index values of the garnet structure for the realization of planar waveguides were first pointed out by Tien et al. in 1972 [6]. In particular the index increase due to gallium substitution in dopedYAG epitaxial waveguides has been demonstrated in a previous paper [7]. Due to the large size of the $\mathrm{Ga}^{3+}$ ion this substitution (for $\mathrm{Al}^{3+}$ ) increases the lattice parameter of the layer and requires an extra compensatory doping by a small rare earth such as lutetium in order to maintain the lattice match with the YAG substrate. Ytterbium-substituted YAG films were grown by means of LPE in a similar fashion to that already reported for neodymium-substituted YAG films [7]. One inch diameter pure YAG substrates with [111] orientation were used. In order to take full advantage of the light confinement offered by a waveguide geometry, layers of only a few microns thickness need to be fabricated but this in turn requires a larger index difference, $\Delta n$, than that produced by the Ybdoping alone.
Therefore, a gallium-lutetium codoping was performed in order to increase the refractive index and at the same time maintain the lattice parameter of the layers.

Just as for the growth of $\mathrm{Nd}$ : YAG, the solvent was composed of $\mathrm{PbO}$ and $\mathrm{B}_{2} \mathrm{O}_{3}$ in the molar ratio of 12 to 1 and an excess of $\mathrm{Al}_{2} \mathrm{O}_{3}$ with respect to $\mathrm{Y}_{2} \mathrm{O}_{3}$ was used in the melts. The saturation temperature $T_{\mathrm{s}}$ depends on the melt composition. Good quality films were prepared at low supersaturation $\Delta T,\left(\Delta T=T_{s}-T_{\text {growth }}\right.$ between 10 and $30^{\circ} \mathrm{C}$ ) at a growth rate up to $1.5 \mu \mathrm{m} /$ minute. Film composition and thus lattice mismatch depend on the growth temperature $T_{\text {growh }}$ and the melt composition. The film thickness depends both on the supersaturation and the growth duration. It was measured by weighing the substrate before and after the growth or by observation of the edge of the layer by optical microscopy.

Table 1 summarises the relevant data from the compositions studied. At first, YAG films with only $\mathrm{Yb}$ substitution were grown at temperatures lower than $1000^{\circ} \mathrm{C}$ with about 3.5 at. \% Yb. We obtain a distribution coefficient for $\mathrm{Yb}$ equal to 1.4 . Then the amount of $\mathrm{Yb}_{2} \mathrm{O}_{3}$ in the melt was increased to reach about 8.5 at. $\%$ in the films. The introduction of a large concentration of gallium required a lattice mismatch compensation with lutetium. Addition of lutetium in the YAG melt leads to an increase of the saturation temperature [7]. Finally, gallium-lutetium codoped films were grown at temperatures around $1000^{\circ} \mathrm{C}$. The distribution coefficients of $\mathrm{Ga}$ and $\mathrm{Lu}$ were calculated to be respectively 0.35 and 2.0 . The last composition appears to be a good compromise between lattice mismatch and index difference for the laser tests. From this melt, films of about $10 \mu \mathrm{m}$ thickness with a low lattice mismatch 
were obtained at a very low supersaturation and hence a small growth rate. As for Nd:YAG waveguides [3], the $\mathrm{Yb}$ : YAG laser layer growth was followed by the growth of an undoped YAG cladding layer grown in an undoped melt. The thickness of this cladding layer was about $20 \mu \mathrm{m}$.

\section{Layer characterization}

\section{I. Lattice mismatch}

$\mathrm{X}$-ray analysis was used to monitor the doping level while the lattice mismatch $\left(\Delta a=a_{\text {substrate }}-a_{\text {layer }}\right)$ was monitored by $\mathrm{X}$-ray diffraction. Change of the garnet lattice parameter, $a_{\text {layer }}$, due to the substitution of yttrium by ytterbium and lutetium are respectively $-0.0007 \AA$ and $-0.0009 \AA$ per at.\% (Fig. 1). Incorporation of gallium required for index adjustment results in an increase in the lattice by $+0.0028 \AA$ per at.\% (Fig. 2). Assuming that these variations are additive and using the compositions determined by $\mathrm{X}$-ray analysis, the calculated lattice mismatch $\Delta a_{\mathrm{c}}$ is found

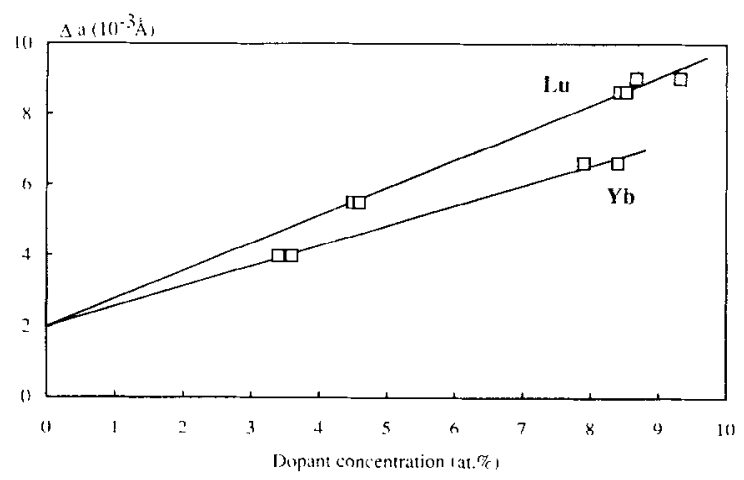

Fig. 1. Lattice mismatch versus dopant concentration for $\mathrm{Lu}$ and $\mathrm{Yb}$ dopings.

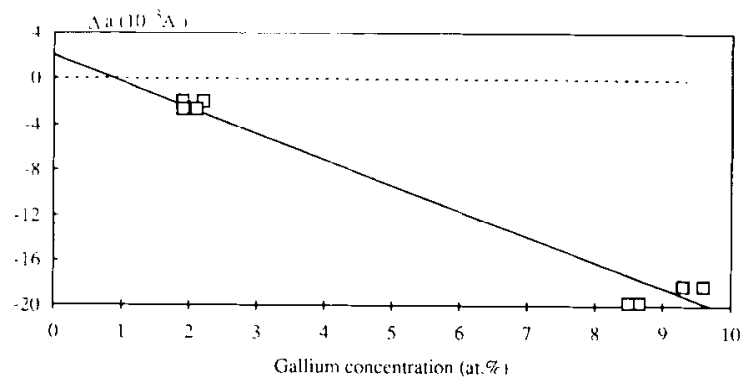

Fig. 2. Lattice mismatch versus dopant concentration for Ga doping.

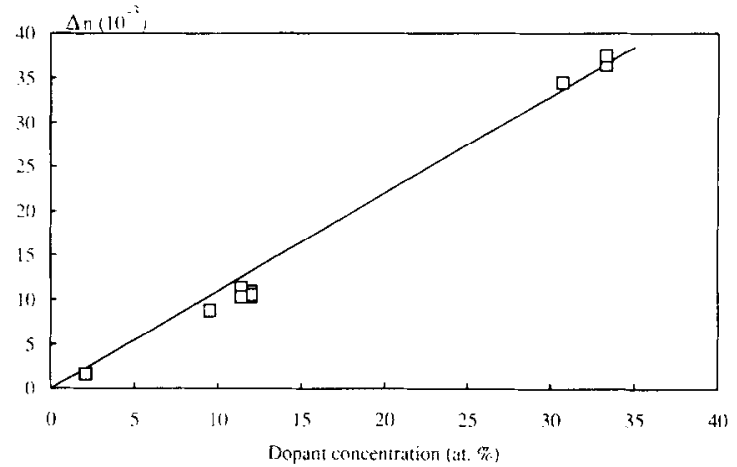

Fig. 3. Film-substrate refractive index difference $\Delta n$ for Ga-doped layers.

to be in agreement with the measured $\Delta a$ (see Table 1). Consequently, an adjustment of the melt composition and of the growth temperature allows the growth of films having a small lattice mismatch and a controlled refractive index difference.

\subsection{Refractive index}

The refractive index difference between the epitaxial layer and the substrate was measured using a classical prism-film coupling technique (dark m-lines). The effect of each dopant on the refractive index was determined by testing films having various compositions. The refractive index increase was in all cases found to be linear with dopant concentration, as shown in Fig. 3 for the case of gallium doping. The slopes of these index variations were measured to be $1.1 \times 10^{-3}$, $0.2 \times 10^{-3}$, and $0.095 \times 10^{-3}$ per at. $\%$ of $\mathrm{Ga}, \mathrm{Yb}$, and $\mathrm{Lu}$, respectively.

\section{Laser results}

\subsection{Experimental set-up}

The guide chosen for assessment of laser performance had a $6 \mu \mathrm{m}$ thick active layer, a cladding layer of $19 \mu \mathrm{m}$ and dopant concentrations of $X_{\mathrm{Yb}}=6.2$ at.\%, $X_{\mathrm{Lu}}=26$ at. $\%$, and $X_{\mathrm{Ga}}=11$ at. $\%$. The refractive index difference was $1.4 \times 10^{-2}$ which results in a waveguide capable of supporting 3 modes at $1 \mu \mathrm{m}$. This guide was cut to a length of $1.6 \mathrm{~mm}$ and its endfaces were polished so as to be parallel to each other and perpendicular to the top surface of the cladding layer. This length is 


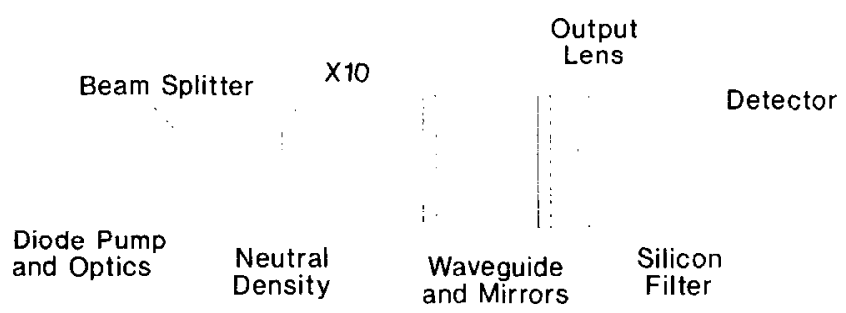

Fig. 4. Experimental set-up for the laser tests.

approximately equal to one absorption length at 968 $\mathrm{nm}$ and is similar to that used in the bulk experiments of Ref. [2]. The plane-parallel laser resonator was made by butting two thin mirrors on the endfaces. These lightweight mirrors were held in place by surface tension using an appropriate liquid [3]. The input mirror had $65 \%$ transmission at the pump wavelength $(968$ $\mathrm{nm}$ ) and $0.6 \%$ transmission at the expected laser wavelength $(1.03 \mu \mathrm{m})$. The output mirror had $71 \%$ reflectivity at $968 \mathrm{~nm}$, providing some feedback of the unabsorbed pump, and $2.3 \%$ transmission at $1.03 \mu \mathrm{m}$. All these transmissions were measured when the mirrors were in contact with YAG.

An InGaAs $1 \mathrm{~W}$ diode-laser (Spectra Diode Laboratories), emitting around $968 \mathrm{~nm}$, was used as the pump. Its output was collimated ( see Fig. 4) by a lens of $6.5 \mathrm{~mm}$ focal length and numerical aperture of 0.62 , and shaped by an anamorphic prism pair. The beam spot size was then matched to the guide dimension using a $\times 10$ microscope objective. The size and shape of the pump waist was observed using an infra-red camera. In the plane perpendicular to the guide the beam was approximately Gaussian with a $1 / \mathrm{e}^{2}$ intensity diameter of $\sim 6 \mu \mathrm{m}$. In the other plane the beam was approximately 'top-hat' in profile with 3 lobes at the peak and a diameter of $\sim 20 \mu \mathrm{m}$.

A silicon plate was used as a spectral filter to separate the residual pump from the waveguide laser beam. The effect of any pump feedback on the performance of the pump diode was monitored using a beam splitter and a silicon photodiode (Fig. 4). Optimum alignment of the pump to the waveguide led to a $30 \%$ increase in the pump power.

\subsection{Results}

The launch efficiency $L$ and the single pass absorption $A$ were measured by comparing the total throughput pump power when the input beam was incident (and optimally launched) on the guide to that when incident on the bulk undoped YAG substrate (using the same focusing arrangement). In the latter case the throughput power $P_{\mathrm{t}}$ is related to the incident power on the focusing objective, $P_{\mathrm{i}}$, by

$P_{\mathrm{t}}=P_{\mathrm{i}} T_{\times 10} T_{\mathrm{F}}^{2}$

and the former case the throughput pump power $P_{\mathrm{t}}$ is

$P_{\mathrm{t}}=P_{\mathrm{i}} T_{\times 10} T_{\mathrm{F}}^{2}(1-L A)$,

where $T_{\times 10}$ is the transmission of the $\times 10$ microscope objective (0.77) and $T_{\mathrm{F}}$ is the transmission of the YAG endfaces. Thus the ratio of the two throughput powers (for the same incident power) is $(1-L A)$ and was measured to be 0.57 . The launch efficiency times single pass absorption product is therefore 0.43 . This would be enough information to calculate the absorbed power threshold $P_{\mathrm{a}}$ from measurements of the incident power threshold if the output mirror did not reflect the pump light, as we would simply use

$P_{\mathrm{a}}=P_{\mathrm{i}} T_{\times 10}\left(1-R_{1}\right) L A$,

where $R_{1}$ is the reflectivity of the input mirror at the pump wavelength (0.35). However the output mirrors used had significant reflectivity at the pump wavelength and so we need to account for unabsorbed pump light being reflected back along the guide adding to the overall absorbed power. In this situation the absorbed power can be related to the incident power by

$$
\begin{aligned}
P_{\mathrm{a}} & =P_{\mathrm{i}} T_{\times 10}\left(1-R_{1}\right) L A\left[1+(1-A) R_{2}\right. \\
& +(1-A)^{2} R_{2} R_{1}+(1-A)^{3} R_{2}^{2} R_{1} \\
& \left.+(1-A)^{4} R_{2}^{2} R_{1}^{2}+\ldots\right],
\end{aligned}
$$

where $R_{2}$ is the reflectivity of the output mirror at the wavelength ( 0.71$)$. In order to evaluate this expression one must know the launch efficiency and absorption separately. However an upper limit for the absorbed power, and hence an upper limit for the absorbed power 


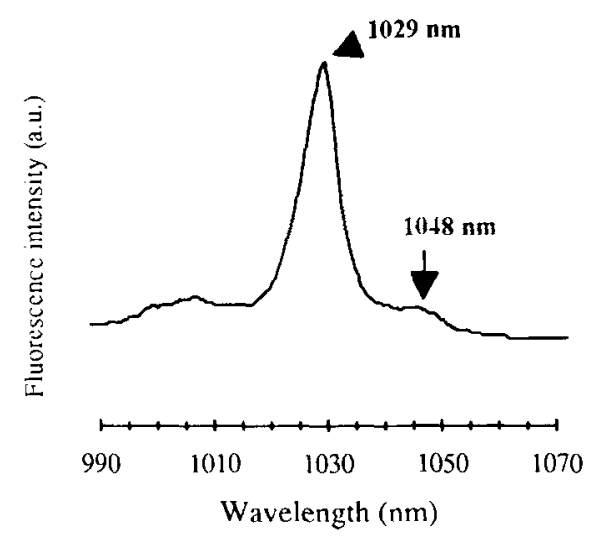

Fig. 5. Fluorescence spectrum of the Yb:Lu:Ga:YAG epitaxial layer.

threshold and a lower limit for the slope efficiency, may be found by assuming $100 \%$ launch efficiency (43\% single pass absorption). Using this assumption we evaluate the above expression to find

$$
\begin{aligned}
P_{\mathrm{a}} & =P_{\mathrm{i}} 0.215(1+0.405 \\
& +0.081+0.033+0.007+\ldots) .
\end{aligned}
$$

This expression carries on with gradually decreasing contributions from the higher order terms. Thus we arrive at the upper limit of $\sim 33 \%$ of the power incident on the $\mathrm{X} 10$ objective being absorbed in the waveguide.

Using the experimental set up shown in Fig. 4 laser emission was observed at either $1029 \mathrm{~nm}$ or $1048 \mathrm{~nm}$, corresponding to two $\mathrm{Yb}^{3+}$ emission peaks as indicated in Fig. 5. The emission cross-section at $1029 \mathrm{~nm}$ (the normal laser line for bulk Yb:YAG [2]) is higher than at $1048 \mathrm{~nm}$. On the other hand, the lower laser level of the $1048 \mathrm{~nm}$ transition is less populated, so the re-absorption losses are less. The mirrors used were of slightly higher reflectivity at $1048 \mathrm{~nm}$ than at $1029 \mathrm{~nm}$ (99.6\% and $98.3 \%$ at $1048 \mathrm{~nm}$ for the input and output mirrors, respectively). Lasing on these two lines has also recently been seen to occur with similar output powers at both wavelengths by other authors [8]. In our particular case we were able to swap between 1029 $\mathrm{nm}$ and $1048 \mathrm{~nm}$ operation by simply moving the waveguide such that we were launching into a different region of the planar guide. This may be due to interferometric effects between the mirror and YAG causing changes in reflectivity in different locations.

The laser tended to work most readily on the 1048 $\mathrm{nm}$ transition. The output power at this wavelength versus absorbed pump power is plotted in Fig. 6 (where we have assumed $100 \%$ launch efficiency). From these results a lower limit on the slope efficiency of $77 \%$ was found. This very high slope efficiency results from the closeness of the pump, $\nu_{\mathrm{p}}$, and signal, $\nu_{\mathrm{s}}$, frequencies $\left(\nu_{\mathrm{s}} / \nu_{\mathrm{p}} \sim 0.9\right)$ and the good overlap between pump and emitted beams which can be realised in waveguides. It is also indicative of the low propagation losses of the waveguide grown by liquid phase epitaxy. The threshold value of $\leq 43 \mathrm{~mW}$ was similar for both lasing lines. The advantage of using waveguides to confine the pump and laser light resulting in high power densities can be seen by comparing this result to the longitudinally diode-pumped bulk $\mathrm{Yb}$ : YAG laser reported elsewhere $[2,9,10]$. Here slope efficiencies of up to $60 \%$ have been obtained but thresholds are in the 100 to 200 $\mathrm{mW}$ range.

The output mode of the laser was observed with an infrared camera and monitor. The output was multimode with a vertical (guided) intensity profile consisting of one main peak and two smaller side lobes, and an approximately Gaussian horizontal intensity profile of $\sim 60 \mu \mathrm{m} 1 / \mathrm{e}^{2}$ intensity radius. The size and shape of the pump mode appeared very close to that of

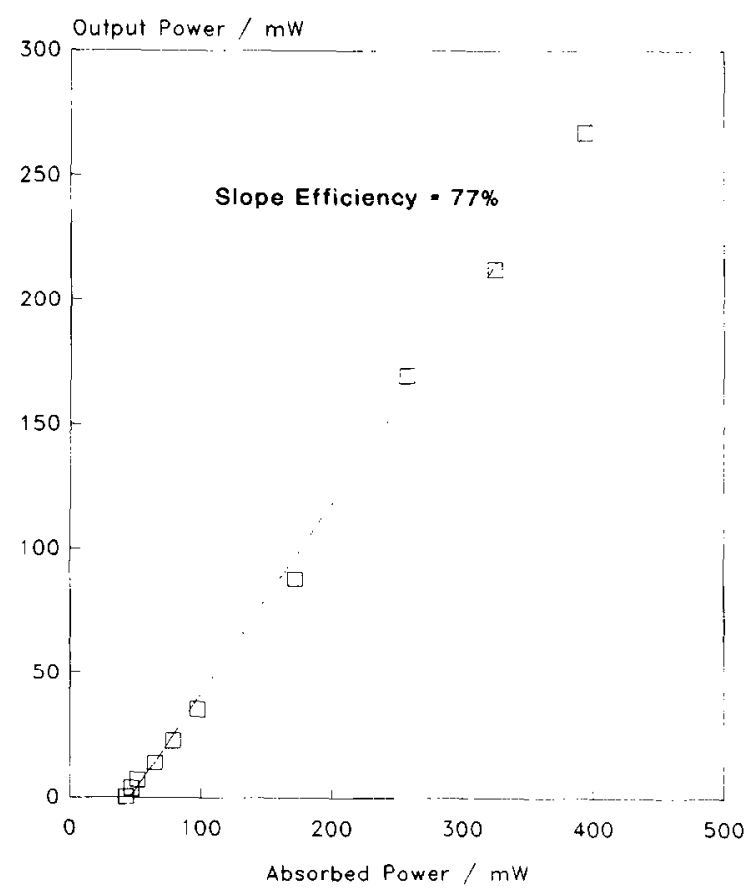

Fig. 6. Output power versus input power for the diode pumped $\mathrm{Yb}$ : YAG waveguide laser at $1048 \mathrm{~nm}$. 
the signal mode except that the horizontal intensity profile was more 'top-hat' in shape.

In order to see whether the laser was performing as expected by theory we have also pumped the $\mathrm{Yb}: \mathrm{YAG}$ with a Ti : sapphire laser. This is an easier case to model as the pump beam is single mode and diffraction limited. The experimental set-up was similar to that described earlier except that the $\mathbf{9 7 0}$ nm diode array (and its beam shaping optics) were replaced by an argon-ion laser pumped $\mathrm{Ti}$ : sapphire laser operating at the shorter pump wavelength of $941 \mathrm{~nm}$. The guide used in this case was longer $(2.4 \mathrm{~mm})$ but was cut and polished from the same epitaxially grown structure as was the $1.6 \mathrm{~mm}$ guide used in the diode-array experiments. Once again laser action could be obtained at $1029 \mathrm{~nm}$ or, more readily, at $1048 \mathrm{~nm}$. In this case the output intensity profile was single mode and approximately Gaussian in both planes. The signal mode had $1 / \mathrm{e}^{2}$ intensity radii of $\sim 2.4 \mu \mathrm{m}$ (guided) and $\sim 41$ $\mu \mathrm{m}$ (non-guided). The guided pump radius was also $\sim 2.4 \mu \mathrm{m}$ and the average spot size in the non-guided plane was calculated to be $95 \mu \mathrm{m}$ [11]. Measurements of the beam quality in terms of the number of times diffraction limited were not made.

The output power at $1048 \mathrm{~nm}$ versus absorbed power (again this is an upper limit for absorbed power found by assuming $100 \%$ launch efficiency) is shown in Fig. 7. It can be seen that the threshold ( $\leq 16 \mathrm{~mW}$ ) is significantly lower than for diode pumping but the slope efficiency is slightly reduced $(\geq 59 \%)$. A theoretical expectation for the absorbed power threshold can be found from [12]

$$
\begin{aligned}
P_{\mathrm{th}} & =\left(\pi h \nu_{\mathrm{p}} / 4 \sigma_{\mathrm{e}} \tau\right)\left(W_{\mathrm{lx}}^{2}+W_{\mathrm{px}}^{2}\right)^{1 / 2} \\
& \times\left(W_{\mathrm{ly}}^{2}+W_{\mathrm{p} y}^{2}\right)^{1 / 2}\left(L+T+2 N_{1}^{0} \sigma_{\mathrm{a}} l\right)
\end{aligned}
$$

where $\nu_{\mathrm{p}}$ is the pump frequency, $\sigma_{\mathrm{e}}$ and $\sigma_{\mathrm{a}}$ are the emission and absorption cross-sections at the laser wavelength, $\tau$ is the fluorescence lifetime, $W$ are the $1 /$ $\mathrm{e}^{2}$ intensity profile radii at the laser (l) and pump (p) wavelengths in the guided $(x)$ and non-guided $(y)$ planes, $L$ is the round-trip propagation loss, $T$ is the total output coupling, $N_{1}^{0}$ is the equilibrium population density of the lower laser level, and $l$ is the length of the crystal. The fluorescence lifetime was measured to be $0.8 \mathrm{~ms}$ which is somewhat less than the $1.16 \mathrm{~ms}$ found in bulk $\mathrm{Yb}$ :YAG [2]. This is probably due to the presence of $\mathrm{Ga}$ and $\mathrm{Lu}$ in the active layer. Assuming

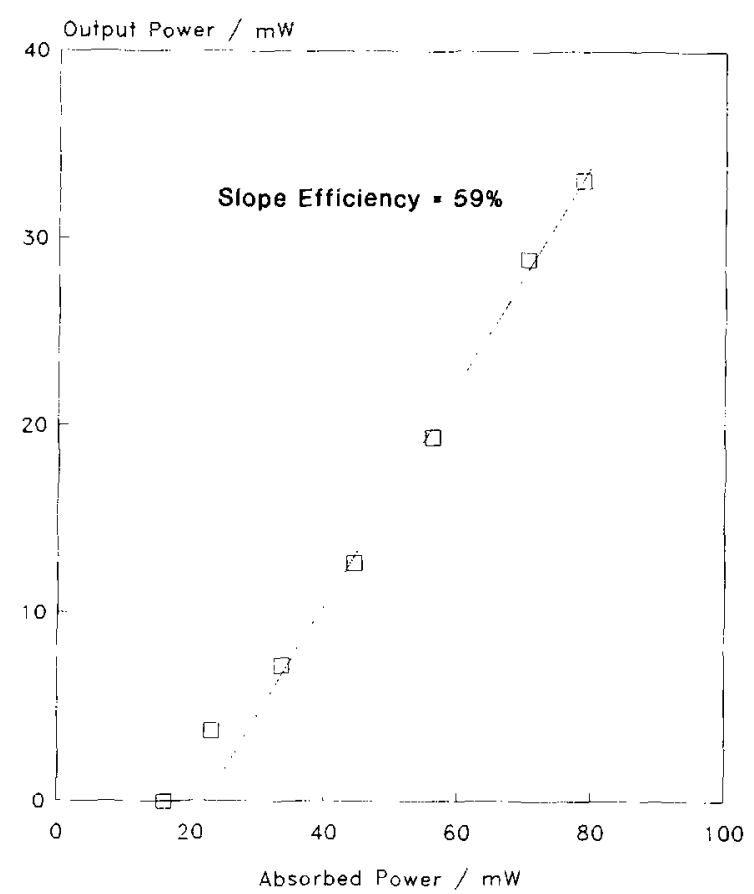

Fig. 7. Output power versus input power for Ti:sapphire pumping of the $1048 \mathrm{~nm}$ emission.

that the emission and absorption cross-sections are the same and equal to the value found in bulk Yb:YAG [2] $\left(1.8 \times 10^{-24} \mathrm{~m}^{2}\right)$, we arrive at an absorbed power threshold for $1029 \mathrm{~nm}$ operation of $P_{\mathrm{th}}=14 \mathrm{~mW}$. In this calculation we have assumed that the propagation losses are much less than the combined transmission and re-absorption losses (i.e. much less than $3 \mathrm{~dB}$ / $\mathrm{cm}$ ). This is in good agreement with the experimentally found value of $\leq 16 \mathrm{~mW}$ (which was the same for $1029 \mathrm{~nm}$ or $1048 \mathrm{~nm}$ lasing). We have only calculated the $1029 \mathrm{~nm}$ threshold as we do not know the crosssections at $1048 \mathrm{~nm}$. Similarly we have not calculated a theoretical slope efficiency at $1048 \mathrm{~nm}$, but as slope efficiencies of $\geq 77 \%$ and $\geq 59 \%$, for $968 \mathrm{~nm}$ diode and $941 \mathrm{~nm} \mathrm{Ti}$ : sapphire pumping respectively, have been observed, it can be deduced that the propagation loss must be much less than the total output coupling (i.e. much less than $0.2 \mathrm{~dB} / \mathrm{cm}$ ). The slope efficiency may be slightly less in the $\mathrm{Ti}$ :sapphire pumped case due to a combination of the lower pump wavelength, the fact that we were not so many times above threshold, and a larger difference between the pump and laser mode sizes in the non-guided direction [12]. 


\section{Conclusion}

We have shown that the laser performance of the quasi-three-level laser $\mathrm{Yb}: \mathrm{YAG}$ is improved in a planar epitaxial waveguide. The waveguide configuration assures a good overlap between the pump and the emitted beams, leading to high slope efficiencies, and the high power densities achievable in waveguides results in the decrease of the intrinsic high threshold of these lasers. Liquid phase epitaxy has been proved to be a good method of producing low loss waveguides suitable for planar lasers. A further order of magnitude reduction in threshold should be obtained if channel waveguides based on these low loss thin films could be produced and fabrication of such guides is currently under investigation. Channel guides could be pumped by single stripe diode lasers for relatively low power applications. For higher output powers planar guides in a longitudinal or side-pumped configuration [13] would be preferred as they are compatible with diode array or diode bar pumping. Extension of this technique to other three level laser dopants in YAG that could benefit from this waveguide geometry, such as $\mathrm{Fr}^{3+}$ and $\mathrm{Tm}^{3+}$, is under way.

\section{Acknowledgements}

The authors are grateful to J.P. Vassali, V. Marty, B. François, G. Rolland and F. Laugier for waveguide preparation and to S. Valette, J.J. Aubert and J.C. Vial for helpful discussions. 'The work carried out at the ORC has been supported by the SERC. This collabo- ration between the ORC and LETI has also been supported by the British Council and the Agence Pour l'Accueil des Personnalités Etrangères via the Alliance Scheme.

\section{References}

[1] A. Eda, K. Shimomura, F. Shimada, K. Yamada and K. Muro, Digest of CLEO 1992 (Opt. Soc. Am., Washington D.C.) communication CWG33.

[2] P. Lacovara, H.K. Choi, C.A. Wang, R.L. Aggarwal and T.Y. Fan, Optics Lett. 16 (1991) 1089.

[3] I. Chartier, B. Ferrand, D. Pelenc, S.J. Field, D.C. Hanna, A.C Large, D.P. Shepherd and A.C. Tropper, Optics Lett. 17 (1991) 810.

[4] D.C. Hanna, A.C. Large, D.P. Shepherd, A.C. Tropper, I Chartier, B. Ferrand and D. Pelenc, Appl. Phys. Lett. 63 (1993) 7.

[5] D.C. Hanna, J.K. Jones, A.C. Large, D.P. Shepherd, A.C. Tropper, P.J. Chandler, M.J. Rodman, P.D. Townsend and L. Zhang, Optics Comm. 99 (1993) 211

[6] P.K. Tien, R.J. Martin, S.H. Wemple and L.J. Varnerin, Appl. Phys. Lett. 21 (1972) 5.

17] B. Ferrand, D. Pelenc, I. Chartier and C. Wyon, J. Cryst. Growth 128 ( I993) 966.

[8] A. Giesen, H. Hügel, A. Voss and K. Wittig, Appl. Phys. B 58 (1994) 365

[9] T.Y. Fan, Optics Lett. 19 (1994) 554

[10] T.Y. Fan, in: Solid State Lasers: New Developments and Applications, eds. M. Inguscio and R. Wallenstein (Plenum, New York, 1993) pp. 189-204.

[11] M.J.F. Digonnet and C.J. Gaeta, Appl. Optics 24 (1985) 333.

[12] W.P. Risk, J. Opt. Soc. Am. B 5 ( 1988 ) 1412.

[13] D.C. Hanna, A.C. Large, D.P. Shepherd, A.C. Tropper, 1 Chartier, B. Ferrand and D. Pelenc, Optics Comm. 91 (1992) 229. 